\title{
The Discourse of the Garment from the Cultural Perspective
}

\author{
Xie Fang 1, a* \\ ${ }^{1}$ Department of Journalism, Complutense University of Madrid, Madrid, Spain. \\ a531805380@qq.com
}

\begin{abstract}
This paper analyzes the behavior of the clothing discourse. For this reason, the analysis will start from the perspective of semiotics, explore the visual discourse of clothing, and reveal the unique structure of clothing. The reason why this paper choose to start with semiotics is that it can treat the discourse of popular culture as a social production system containing cultural experience. Here, relying on semiotic theorists such as Saussure, F and Barthes, R, clothing is constructed as a visual sign within discourse framework, so as to explore its social meaning in the complexity of the construction and deconstruction of sign, and show the dynamics of clothing discourse.
\end{abstract}

Keywords: clothing, sign, culture, discourse

\section{文化视阈下的服装话语}

解芳 $1, \mathrm{a}^{*}$

1 马德里康普顿斯大学新闻系, 马德里, 西班牙
a531805380@qq.com

摘要:

本文是对服装中的话语行为进行分析。为此, 分析将从符号学的角度开始, 探索服装的视觉话语, 揭示服装自 身独特的结构。之所以选择从符号学入手是因为它能够将大众文化中的话语作为包含文化经验的社会生产体 系来处理。在这里, 依靠诸如索绪尔, 罗兰巴特等理论家的符号学理论, 将服装构建成为话语框架内的视觉符 号，从而在符号的构建与解构的复杂性中探索其社会意义，显示服装话语的动态性。

关键词: 服装, 符号, 文化, 话语

\section{1. 符号与服装}

在《民生主义》中，孙中山首次提出了 “衣食住 行” 的概念, 从社会结构的发展情境来看, 以衣为首 是充分显示了人与动物的区别, 体现了人类文明。相 比于食住行三项, 服装的出现与发展过程与人类文明 的进步几乎是同步的, 这就说明, 衣, 是一种人类发 展的一个缩影。而关于服装的研究, 从来都不是单单 属于设计学和制造业等领域, 它更多的是展示了一种 文化。因此, 探索文化视阈下的服装话语就要求我们 不能将服装单单作为一种物质实体, 而是将其当作一 个符号置于文化的领域。
瑞士结构主义语言学家索绪尔曾在《普通语言学 教程》一书里提出了符号的概念, 他将语言称作为符 号系统, 并指出语言符号的连接不只是事物与名称的 拼接, 而是概念与音响形象的结合体。因此, 为了更 好的研究语言符号的形象与概念, 他将每一个符号划 分出了能指和所指两个部分。同时, 他还强调语言符 号任意性的特点, 也就是说, 符号的能指和所指的结 合就能形成了一个任意的实体。

索绪尔的语言符号学理论改变了语言与现实的 关系, 语言不再是现实世界的再现, 它有着自己的结 构, 并且通过这种结构, 构建出语言的现实意义。然 而, 符号学作为一门研究意义的学科, 它首先是建在 大量的话语的基础之上, 这种基于语言学中的话语符 
号学被当作了广义符号学的先行者, 随着符号学的逐 步发展, 它开始涉足了研究大众话语。

这种符号学的转向要求分析者从更复杂的角度 看待这些大众话语。因此, 在索绪尔理论的影响下, 罗兰巴特开始拓展符号学的研究领域, 他认为符号学 不应仅仅是适用于自然语言, 更应该适用于解释出现 在日常生活中的所有现象。他在自然语言范式之上集 中解释了各种文化现象的意义, 也就是说, 他为这些 社会话语提供了一个符号学的分析框架。

在巴特的符号学原理之下, 所有事物都将被重新 定义为具有意义的符号。这样一来, 在大众文化所流 传的社会话语材料中, 必然会存在多种不同于语言元 素的其他元素, 而视觉元素就是其中的代表。随着具 有文化意义的各种元素的引入, 符号学也就会出现了 各种各样的分支。而在这里, 我们主要关注服装, 并 将其作为一种视觉元素, 通过符号学的视角去解释它 们。巴特将符号学作为一种方法论, 是用于对一切事 物的一种在思考, 在 1967 年巴特发表的《流行体系》 一书中, 他将服装比做一个符号, 他指出服装的功能 不仅仅在于提供一种复制现实的样式, 更重要的把服 装作为一种意义加以广泛的传播。所以, 服装是以视 觉交流为基础的一种社会话语实践, 它揭示了社会本 身的反映性的表示, 以及印在其上的各种美学概念和 社会文化习惯。

\section{2. 服装话语系统的构建}

《流行体系》一书是巴特对于《符号学原理》的 一种实践, 他从符号学角度构建了一种全新的服装体 系。他将自然语言的理论运用于在服装的研究上, 并 以此探究服装的意义。服装从最初保暖遮体的功能, 演变成为了一种审美和流行的趋向。它受到了来自时 代, 社会, 文化, 宗教, 政治, 经济等不同因素的影 响, 并且在这些因素的共同影响下形成了一种服装特 有的符号体系。

根据巴特的理论, 服装系统分为直接意指层与含 蓄意指层, 而每层结构都有与其对应的表达层和内容 层。在服装体系的意义构建的过程中, 历史的阐述和 符号记忆的介入, 使得服装成为了叙事意义的实体, 其意义随着时间而产生和再现。因此, 服装作为一个 视觉符号想要产生意义就必须与一个已知对象相关 联。可以说, 服装的视觉意义的形成过程是通过建 立意义的关系来完成的, 而这种意义的构建不仅仅是 由一个社会主体完成的, 而是由两个主体共同参与而 形成的。即话语的发出者和话语的接受者。只有在二 者的共同的作用下, 意义才能得以展现。

从推论的角度理解服装意义解释过程, 就是从服 装话语分析中找到其理论空间。在这种分析中, 服装 作为交际主体而不是依靠简单的符码进行上下文的 推理, 而是从一开始就要对接收者的反应进行预期。 在这个系统中, 任何一件服装的能指与所指的结合都 离不开符码。由于系统不是单层的结构, 而是双层的,
因此, 处于直接意指层的符码是一种“语言学符码”, 而处于内涵意指层的符码必定是 “文化符码”。在这 个双层符号结构之中, 服装话语是一种用于信息的发 出者与信息接收者之间的相互交流的符号系统，而所 有的意义的实现都要依赖于这些符码。因此, 服装话 语是由一套符码构成, 接收者根据符码对服装进行解 释, 在解释的过程中, 服装的意义得以丰富。而这些 意义的多样性是由服装自身生成的东西。可以说服装 话语系统是寻求一种与社会和文化动态相关的沟通 过程。

服装是一种元文化, 它能够借助社会历史所形成 文化背景作为一种传播途径，促进其自身的传播。因 此, 服装的意义不仅限于它自身产生的, 更多的是靠 话语而实现的。话语的存在是为了用于沟通交流, 这 是社会生产, 交换, 协商的结果。因此, 在符号的领 域下, 将话语理解成空间, 在这些空间中, 服装将世 界构造为客体, 并将自身当作主体, 通过这些话语, 人们可以接近服装主体的构成, 毕竟分析服装话语的 目的就是要了解其文本意义已经它的运作形式。这样 一来, 在服装话语材料引入了具有多种文化的视觉元 素, 丰富了服装的意义。话语可以被解释为一种构建 社会意识的空间, 在这一空间中, 服装的陈述得以再 现, 这些陈述构建并合法化了服装所代表的现实。

在这一过程中, 信息的发出者经过传播渠道将信 息传递给接收者, 这是一个人类文化与知识传递的必 经途径。话语一词因而被用来指代符号过程, 是服装 从根本上的一种交流。这就是话语与表达领域内的服 装符号直接相关的方式。一般来说, 视觉符号学可以 分析话语领域中的文本, 包括文化意义, 社会实践, 以及权利关系等, 并在这些过程相互作用, 而后构建 了影响文化和社会历史背景的视觉动力。

可以说, 语言空间促进或共享了非语言元素, 并 且可以通过这些视觉提要中进行解读, 也就是说, 话 语以一种综合的方式提出关系, 向人们展示着各种异 类元素。视觉作为一种感知行为的体验, 将人们带入 了虚构的领域。

现如今的当下生活, 服装成为了表达时尚的一种 社会机制, 其存在与传播紧密相连。由于服装自身的 符号结构, 它可以被当作为一个意义的制造机, 充当 着多种多样的信息源头。正如 20 世纪 20 年代旗袍, 它在传统的宽大袍服的基础上, 经历了一系列的变化, 逐步形成了我们如今所见的旗袍样式。可以说旗袍见 证了中国一个多世纪的时尚演变, 这也标志了封建政 权结束后民主思想的萌芽, 服装的变化在时尚界留下 了印记, 也由此向我们展示了东西方文化融合的社会 现实。

\section{3. 服装话语系统的应用}

现在, 服装话语可以被认为社会话语之间的或者 是实现这种话语沟通的中间场所。它将视觉文本置于 社会话语实践的框架内, 在范式与语义内进行进行阐 
述, 这也使得服装超出了固有的文本性的框架。服装 话语作为话语的一种分支, 再加之符号学的概念的引 入, 不仅将服装置于特定的表达空间，同时也将服装 集中起来得到社会规范与认定。

服装作为一种视觉符号, 自古以来一直是展现人 的外在形象的一种载体。在不同的场合中, 它依赖于 不同的符码展示着不同的意义。这些意义并不是一成 不变的, 而是随着时间的推移, 慢慢地发生着改变。

在封建时期的中国, 服装是区分人类等级地位的 一种手段。材质, 款式与颜色是服装组成的三大部分。 一般来说, 富贵人家多以丝织品为主, 而广大的百姓 则是身穿粗布麻衣。在款式上相对单一, 在明末之前 都以汉服为主, 直至清王朝的出现袍服才逐渐走进大 众视线。而颜色作为服装的重要组成部分, 自始至终 都起着不可磨灭的作用。黄色作为封建时期的权力的 代表色, 象征了大地, 因此, 黄色被赋予了占有土地 的意味, 宣誓了统治者的绝对统治地位, 所以只有历 朝历代的统治者才可以穿戴黄色。黄色的服装也因此 成为了象征统治者的特权的一种标志。这一特点在很 多中国的影视作品中得以体现, 以张艺谋导演的满城 尽带黄金甲为代表, 整部电影的黄色服装给人以眼花 缭乱之感, 无论皇帝的服装款式, 材质如何变化, 都 不能改变它的核心色彩-黄色, 由此凸显了皇帝的至 高无上的统治地位以及不可侵犯的威严。

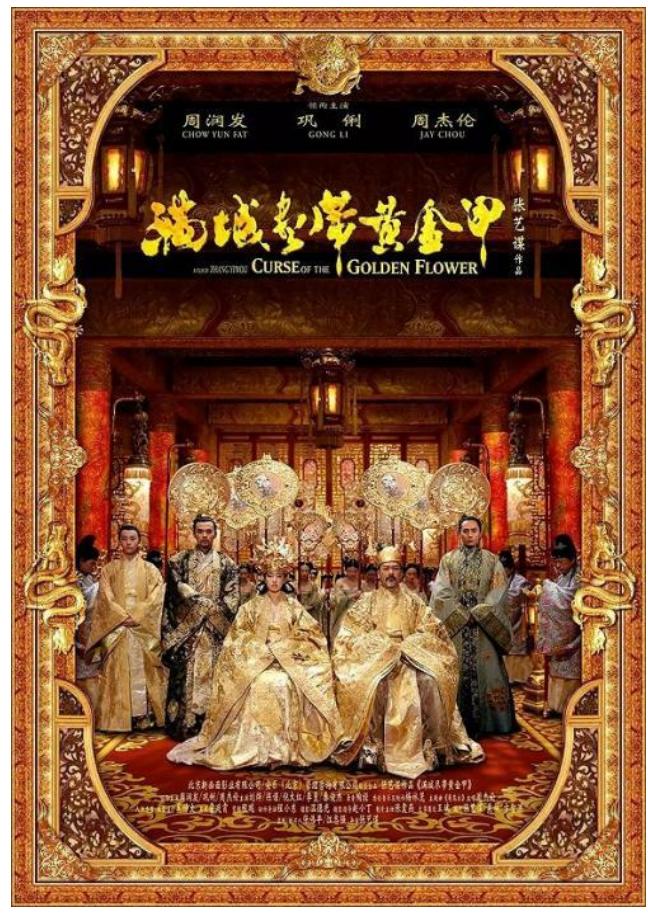

图 1 满城尽带黄金甲海报

随着封建制度的瓦解, 民主思想的兴起, 黄色 不再是帝王的专属, 大街小巷的人们也都开始穿起了 黄色的衣服。中国的服装款式也从传统单一的款式转 向多样化的发展。以中山装为例, 这套以孙中山先生 设计的中式西装, 一问世就受到中国男性的追捧。这 种胸前的五颗扣子, 四个口袋, 两袖口每侧三颗扣子
的设计一直沿用至今, 成为经典。他的每个细节的设 计都有其独特的意义, 中山装的设计理念中融入了五 权宪法, 三民主义以及中国传统的 “礼义廉耻” 的四 个维度。既传承了中国的传统文化思想, 同时又融合 了新的民主思想。在大部分人的心里, 中山装的诞生 可以说是中西文化融合的典范, 它凭借其特殊的文化 内涵和设定的历史时期转变成为了革命者的象征。然 而，随着时间的流逝，中山装逐渐成为了具有中华民 族的代表性服装, 它更多的是代表了多元化文化的融 合, 着重于体现中国民族的价值取向, 审美情趣和文 化认同。此时的中山装, 更多的是被赋予了政治性的 内涵。在 2014 年, 习主席身穿一套经过改良的中山 装出席了荷兰国王举行的国宴。与传统的西装相比, 这套经过特意改版的中山装, 更具深意。在传统中山 装的样式上增添了放置口袋巾的设计, 既使得中国传 统服装的样式得以保留, 同时也融合了西方的文化元 素。这样的修改, 延续了中山装本身的精神内涵, 同 时也特意表达出对西方文化的尊重。中山装利用对外 传播的途径, 成为了国家形象和软实力的代表, 展现 了对本国文化的高度自信感, 对文化外交和经济外交 有着至关重要的影响。

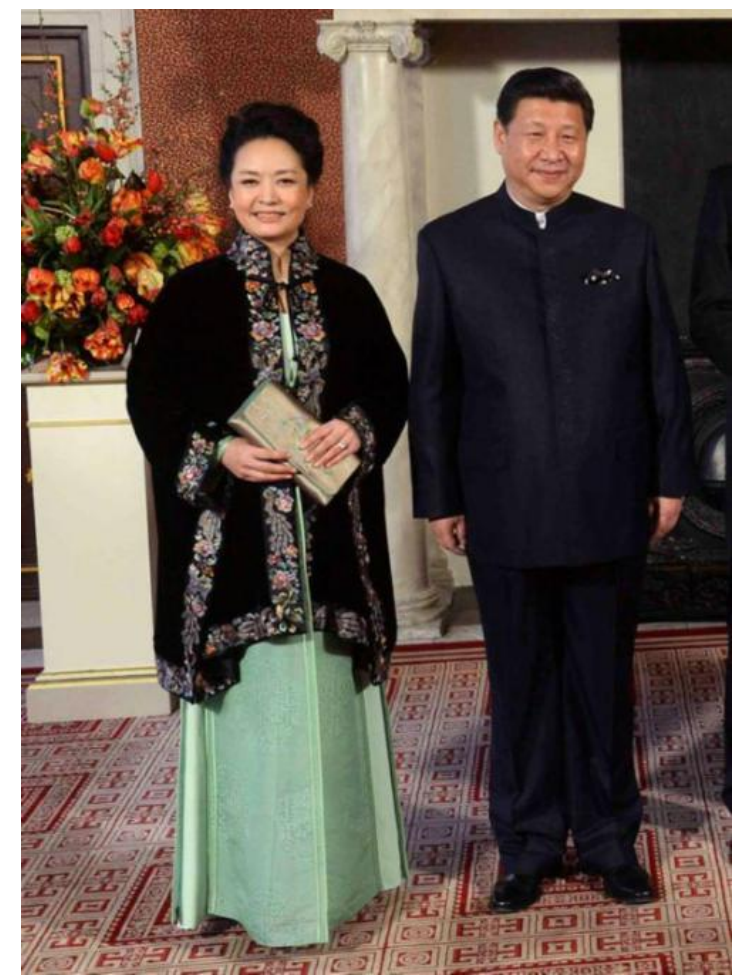

图 2 习近平主席出席荷兰国宴的着装

随着科技技术的发展, 新媒体的广泛应用, 人民 生活条件的提升，服装的款式也在不停的更替，人们 不再以一成不变的方式对服装的各个方面进行严格 的限制, 由此应运而生了时尚的观念。随着后现代的 到来, 人们的主观意识的增强, 展现了不同的穿着的 方式。正如齐美尔指出的社会的变革对时尚产生的巨 大影响一样, 时代变化越快, 时尚的更替也就越快。 在这个互联网的时代, 信息的飞速传播, 也给服装带 来的巨大影响力, 其快速的发展, 带动了相关产业, 
扩大了不同文化资源的影响, 对于社会经济的推动有 着潜在但又深远的意义。

\section{4. 结语}

随着服装的不断发展, 我们正在试图在涉及视觉 话语中, 寻求服装与意义之间新的联系。正是由于符 号学的介入, 才使得人们窥视到服装的文化意义, 并 实现了意义的储存与交流的功能。

服装被认定为符号, 经过聚合形成一种整体, 从 符号学的角度来看, 服装话语的构建过程是在多个层 面多个维度同时前进的, 但其中必不可少的就是符号, 文化和传播，三者之间建立了一个极其复杂的关系， 需要依靠符号学的方法论, 通过文化符码的编码与解 码在通过特定的传播途径, 才能得以理解。在这一系 统中, 服装的每个方面都得以关注。在这里, 服装不 再仅仅是传统意义上的简单的穿着, 更是一种文化的 彰显。

在文化融合日趋激烈的世界中，现代中国服装， 从起源到现在, 历史悠久, 随着国际社会的加入, 中 国服装必定成为世界时尚的一个独特的部分, 与其他 文化并存, 并产生新的意义, 这无疑成为一种不可逆 转的趋势。

\section{REFERENCES}

[1] Saussure, F (1959) Course in General Linguistics. The Philosophical Library, New York City.

[2] Barthes, R (1990) The Fashion System, University of California Press, California.

[3] Eco, U (2005) On Literature, Harvest Books, New York.

[4] Simmel, G (2017) The philosophy of fashion, Guangdong Flower City Publishing House, Guangdong. Guangdong Flower City Publishing House. 